
Physics

\title{
On Modulated NLS-Ermakov Systems
}

Colin Rogers, Kwok Chow

To cite this article: Colin Rogers, Kwok Chow (2017) On Modulated NLS-Ermakov Systems, Journal of Nonlinear Mathematical Physics 24: Supplement 1, 61-74, DOI: https://doi.org/10.1080/14029251.2017.1418054

To link to this article: https://doi.org/10.1080/14029251.2017.1418054

Published online: 04 January 2021 


\title{
On Modulated NLS-Ermakov Systems
}

\author{
Colin Rogers \\ School of Mathematics and Statistics, \\ The University of New South Wales, \\ Sydney, NSW2052, Australia \\ c.rogers@unsw.edu.au \\ Kwok Chow \\ Department of Mechanical Engineering, \\ The University of Hong Kong, \\ Hong Kong \\ kwchow@hku.hk
}

Received 7 September 2017

Accepted 6 October 2017

\begin{abstract}
Spatial modulated coupled nonlinear Schrödinger systems with symmetry reduction to integrable Ermakov and Ermakov-Painlevé subsystems are investigated.
\end{abstract}

\section{Introduction}

Physical systems with spatial modulation arise in a range of important contexts. In classical continuum mechanics, they occur 'inter alia' in elastodynamics, visco-elastodynamics and in crack and loading boundary value problems in the elastostatics of inhomogeneous media (see e.g. [2,12,13,20] and work cited therein). Nonlinear Schrödinger (NLS) equations with modulation have, in recent years, been shown to have importance in the theory of Bose-Einstein condensates and in nonlinear optics, in particular, in the area of soliton management [6,7,22,44,48-50]. Nonlinear coupled systems of Ermakov-Ray-Reid type with origin in classical work in [16] and subsequently developed in [24-26] likewise have extensive physical applications (see e.g. $[1,14,15,17-19,27-31,43,46]$ ).

In recent work, prototype integrable Ermakov-Painlevé II and Ermakov-Painlevé IV systems have been derived as symmetry reductions of Manakov-type and resonant NLS systems of physical interest $[32,33]$. Here, canonical reduction of modulated NLS-Ermakov systems is investigated.

\section{Modulated Nonic NLS Systems: Ermakov Reduction}

Localised and periodic wave patterns admitted by single component unmodulated nonic NLS equations have recently been isolated by use of key invariants of motion in [9]. Here, coupled spatially modulated nonic NLS-Ermakov systems are investigated of the type

$$
\begin{gathered}
i \Phi_{t}+\Phi_{x x}-\left[\Omega(x)+\Sigma_{k=1}^{4} h_{k}(x)\left(|\Phi|^{2}+|\Psi|^{2}\right)^{k}\right] \Phi=\frac{1}{|\Phi|^{3}|\Psi|} S\left(\left|\frac{\Psi}{\Phi}\right|\right) \Phi \\
i \Psi_{t}+\Psi_{x x}-\left[\Omega(x)+\Sigma_{k=1}^{4} h_{k}(x)\left(|\Phi|^{2}+|\Psi|^{2}\right)^{k}\right] \Psi=\frac{1}{|\Psi|^{3}|\Phi|} T\left(\left|\frac{\Phi}{\Psi}\right|\right) \Psi . \\
\text { Co-published by Atlantis Press and Taylor \& Francis } \\
\text { Copyright: the authors }
\end{gathered}
$$


In the sequel, we introduce the wave packet ansätze

$$
\Phi=q_{1}(x) \exp \left[-i \mu t+i r_{1}(x)\right] \quad, \quad \Psi=q_{2}(x) \exp \left[-i \mu t+i r_{2}(x)\right]
$$

and proceed with modulations

$$
h_{k}(x)=H_{k}\left(\frac{q_{1}}{q_{2}}\right) / \rho^{4+2 k} \quad, \quad k=1, \ldots, 4 .
$$

It is remarked that, symmetry reduction of an unmodulated Manakov system under such representations has been previously used to isolated periodic and quasi-periodic solutions by Christiansen $e t$ al in [10].

Insertion of (2.2) into (2.1) produces the coupled nonlinear system

$$
\begin{gathered}
q_{1 x x}+\mu q_{1}-r_{1 x}^{2} q_{1}-\left[\Omega(x)+\Sigma_{k=1}^{4} h_{k}(x)\left(q_{1}^{2}+q_{2}^{2}\right)^{k}\right] q_{1}=\frac{1}{q_{1}^{2} q_{2}} S\left(\frac{q_{2}}{q_{1}}\right), \\
q_{2 x x}+\mu q_{2}-r_{2 x}^{2} q_{2}-\left[\Omega(x)+\Sigma_{k=1}^{4} h_{k}(x)\left(q_{1}^{2}+q_{2}^{2}\right)^{k}\right] q_{2}=\frac{1}{q_{1} q_{2}^{2}} T\left(\frac{q_{1}}{q_{2}}\right)
\end{gathered}
$$

together with

$$
2 r_{1 x} q_{1 x}+r_{1 x x} q_{1}=0 \quad, \quad 2 r_{2 x} q_{2 x}+r_{2 x x} q_{2}=0
$$

whence

$$
r_{1 x}=k_{\mathrm{I}} / q_{1}^{2} \quad, \quad r_{2 x}=k_{\mathrm{II}} / q_{2}^{2}
$$

where $k_{\mathrm{I}}, k_{\mathrm{II}}$ are arbitrary constants of integration.

Substitution of the modulations (2.3) and the relations (2.6) into (2.4) now yields

$$
\begin{aligned}
q_{1 x x}+[\mu-\Omega(x)] q_{1}= & \frac{1}{q_{1}^{2} q_{2}} S^{*}\left(\frac{q_{2}}{q_{1}}\right)+\frac{q_{1}}{\rho^{4}} \Sigma_{k=1}^{4} H_{k}\left(\frac{q_{1}}{q_{2}}\right)\left[\left(\frac{q_{1}}{\rho}\right)^{2}+\left(\frac{q_{2}}{\rho}\right)^{2}\right]^{k}, \\
& :=\frac{q_{1}}{q_{2}^{4}} \phi\left(q_{1} / q_{2}\right)+\frac{q_{1}}{\rho^{4}} \psi\left(q_{1} / \rho, q_{2} / \rho\right) \\
q_{2 x x}+[\mu-\Omega(x)] q_{2}= & \frac{1}{q_{2}^{2} q_{1}} T^{*}\left(\frac{q_{1}}{q_{2}}\right)+\frac{q_{2}}{\rho^{4}} \Sigma_{k=1}^{4} H_{k}\left(\frac{q_{1}}{q_{2}}\right)\left[\left(\frac{q_{1}}{\rho}\right)^{2}+\left(\frac{q_{2}}{\rho}\right)^{2}\right]^{k}, \\
& :=\frac{q_{2}}{q_{1}^{4}} \chi\left(q_{1} / q_{2}\right)+\frac{q_{2}}{\rho^{4}} \psi\left(q_{1} / \rho, q_{2} / \rho\right)
\end{aligned}
$$

where

$$
S^{*}\left(q_{2} / q_{1}\right)=S\left(q_{2} / q_{1}\right)+k_{\mathrm{I}}^{2}\left(q_{2} / q_{1}\right) \quad, \quad T^{*}\left(q_{1} / q_{2}\right)=T\left(q_{1} / q_{2}\right)+k_{\mathrm{II}}^{2}\left(q_{1} / q_{2}\right) .
$$

If the above pair of coupled nonlinear equations is augmented by the generalised Ermakov equation

$$
\rho_{x x}+[\mu-\Omega(x)] \rho=\frac{1}{\rho^{3}} \psi\left(q_{1} / \rho, q_{2} / \rho\right)
$$

then the resultant triad constitutes a three-component Ermakov system as originally introduced in a multi-layer hydrodynamic context in [34]. 
On introduction of the change of dependent and independent variables according to

$$
\begin{gathered}
q_{1}=q_{1}^{*}(z) \phi^{*} \quad, \quad q_{2}=q_{2}^{*}(z) \phi^{*}, \\
\rho=\rho^{*}(z) \phi^{*},
\end{gathered}
$$

with

$$
z=\psi^{*} / \phi^{*}
$$

where $\phi^{*}, \psi^{*}$ are linearly independent solutions with unit Wronskian of

$$
\Delta_{x x}+[\mu-\Omega(x)] \Delta=0
$$

the non-autonomous triad determined by (2.7)-(2.9) is reduced to the corresponding autonomous form

$$
\begin{gathered}
q_{1 z z}^{*}=\frac{q_{1}^{*}}{q_{2}^{* 4}} \phi\left(q_{1}^{*} / q_{2}^{*}\right)+\frac{q_{1}^{*}}{\rho^{* 4}} \psi\left(q_{1}^{*} / \rho^{*}, q_{2}^{*} / \rho^{*}\right), \\
q_{2 z z}^{*}=\frac{q_{2}^{*}}{q_{1}^{* 4}} \chi\left(q_{1}^{*} / q_{2}^{*}\right)+\frac{q_{2}^{*}}{\rho^{* 4}} \psi\left(q_{1}^{*} / \rho^{*}, q_{2}^{*} / \rho^{*}\right), \\
\rho_{z z}^{*}=\frac{1}{\rho^{* 3}} \psi\left(q_{1}^{*} / \rho^{*}, q_{2}^{*} / \rho^{*}\right) .
\end{gathered}
$$

Thus, it is seen that

$$
\begin{aligned}
& \rho^{*} q_{1 z z}^{*}-q_{1}^{*} \rho_{z z}^{*}=\frac{q_{1}^{*} \rho^{*}}{q_{2}^{* 4}} \phi\left(q_{1}^{*} / q_{2}^{*}\right), \\
& \rho^{*} q_{2 z z}^{*}-q_{2}^{*} \rho_{z z}^{*}=\frac{q_{2}^{*} \rho^{*}}{q_{1}^{* 4}} \chi\left(q_{1}^{*} / q_{2}^{*}\right),
\end{aligned}
$$

whence on setting

$$
u^{*}=q_{1}^{*} / \rho^{*}=q_{1} / \rho \quad, \quad v^{*}=q_{2}^{*} / \rho^{*}=q_{2} / \rho,
$$

and introduction of a reciprocal-type transformation

$$
d z^{*}=\frac{1}{\rho^{* 2}} d z \quad, \quad \rho^{* *}=\frac{1}{\rho^{*}},
$$

(2.14) becomes an autonomous two-component Ermakov-Ray-Reid system

$$
u_{z^{*} z^{*}}^{*}=\frac{1}{u^{* 2} v^{*}} S^{*}\left(\frac{v^{*}}{u^{*}}\right) \quad, \quad v_{z^{*} z^{*}}^{*}=\frac{1}{v^{* 2} u^{*}} T^{*}\left(\frac{u^{*}}{v^{*}}\right),
$$

while

$$
\rho_{z^{*} z^{*}}^{* *}+\psi\left(u^{*}, v^{*}\right) \rho^{* *}=0 .
$$

The latter linear equation in $\rho^{* *}$ becomes determinate once the Ermakov variables $u^{*}, v^{*}$ have been obtained via the system (2.17). 
The Ermakov-Ray-Reid system (2.17) is Hamiltonian with

$$
\frac{1}{u^{* 2} v^{*}} S^{*}\left(\frac{v^{*}}{u^{*}}\right)=\frac{\partial V}{\partial u^{*}} \quad, \quad \frac{1}{v^{* 2} u^{*}} T^{*}\left(\frac{u^{*}}{v^{*}}\right)=\frac{\partial V}{\partial v^{*}}
$$

provided it adopts the form [27]

$$
u_{z^{*} z^{*}}^{*}=\frac{2}{u^{* 3}} J^{*}\left(v^{*} / u^{*}\right)+\frac{v^{*}}{u^{* 4}} J^{*^{\prime}}\left(v^{*} / u^{*}\right) \quad, \quad v_{z^{*} z^{*}}^{*}=-\frac{1}{u^{* 3}} J^{*}\left(v^{*} / u^{*}\right)
$$

with Ermakov invariant

$$
\mathscr{E}^{*}=\frac{1}{2}\left(v^{*} u_{z^{*}}^{*}-u^{*} v_{z^{*}}^{*}\right)^{2}+\left(\frac{u^{* 2}+v^{* 2}}{u^{* 2}}\right) J^{*}\left(v^{*} / u^{*}\right)
$$

and Hamiltonian

$$
\mathscr{H}^{*}=\frac{1}{2}\left(u_{z^{*}}^{* 2}+v_{z^{*}}^{*}\right)^{2}+\frac{1}{u^{* 2}} J^{*}\left(v^{*} / u^{*}\right)
$$

This pair of invariant relations in $\mathscr{E}^{*}$ and $\mathscr{H}^{*}$ may be used to established the integrability of the Ermakov-Ray-Reid system (2.20) in an algorithmic manner as described in [27]. Three-component Ermakov systems of the type (2.13) have been investigated in detail in [34]. Novel connections of such Ermakov systems and 3-body problems have been recently established in [35].

In [6,7], a symmetry reduction of a single component NLS equation with modulation $h(x) \sim \rho^{-6}$ of the cubic nonlinear term was considered where $\rho$ is driven by

$$
\rho_{x x}+[\mu-\Omega(x)] \rho=\frac{k_{\mathrm{III}}}{\rho^{3}},
$$

namely, the classical Ermakov equation. Modulated single-component cubic NLS equations associated with standard Ermakov-Ray-Reid systems have recently been investigated in [36]. Here, in the present context of the coupled nonic NLS system (2.1), the class of modulations

$$
h_{k}(x)=c_{k} / \rho^{4+2 k} \quad, \quad k=1, \ldots, 4
$$

is next adopted wherein $\rho$ is constrained by (2.23). The symmetry reduction of the corresponding modulated system (2.1) via (2.2) then yields

$$
\begin{aligned}
& q_{1 x x}+[\mu-\Omega(x)] q_{1}=\frac{1}{q_{1}^{2} q_{2}} S\left(\frac{q_{2}}{q_{1}}\right)+\frac{k_{\mathrm{I}}^{2}}{q_{1}^{3}}+\frac{q_{1}}{\rho^{4}} \Sigma_{k=1}^{4} c_{k}\left[\left(\frac{q_{1}}{\rho}\right)^{2}+\left(\frac{q_{2}}{\rho}\right)^{2}\right]^{k}, \\
& q_{2 x x}+[\mu-\Omega(x)] q_{2}=\frac{1}{q_{2}^{2} q_{1}} T\left(\frac{q_{1}}{q_{2}}\right)+\frac{k_{\mathrm{II}}^{2}}{q_{2}^{3}}+\frac{q_{2}}{\rho^{4}} \Sigma_{k=1}^{4} c_{k}\left[\left(\frac{q_{1}}{\rho}\right)^{2}+\left(\frac{q_{2}}{\rho}\right)^{2}\right]^{k} .
\end{aligned}
$$

Combination with the Ermakov equation (2.23) now shows that

$$
\begin{aligned}
& \rho q_{1 x x}-q_{1} \rho_{x x}=\frac{\rho}{q_{1}^{2} q_{2}} S\left(\frac{q_{2}}{q_{1}}\right)+\frac{\rho k_{\mathrm{I}}^{2}}{q_{1}^{3}}-\frac{k_{\mathrm{III}} q_{1}}{\rho^{3}}+\frac{q_{1}}{\rho^{3}} \Sigma_{k=1}^{4} c_{k}\left[\left(\frac{q_{1}}{\rho}\right)^{2}+\left(\frac{q_{2}}{\rho}\right)^{2}\right]^{k}, \\
& \rho q_{2 x x}-q_{2} \rho_{x x}=\frac{\rho}{q_{2}^{2} q_{1}} T\left(\frac{q_{1}}{q_{2}}\right)+\frac{\rho k_{\mathrm{II}}^{2}}{q_{2}^{3}}-\frac{k_{\mathrm{III}} q_{2}}{\rho^{3}}+\frac{q_{2}}{\rho^{3}} \Sigma_{k=1}^{4} c_{k}\left[\left(\frac{q_{1}}{\rho}\right)^{2}+\left(\frac{q_{2}}{\rho}\right)^{2}\right]^{k}
\end{aligned}
$$


whence, on setting

$$
\begin{gathered}
u=q_{1} / \rho \quad, \quad v=q_{2} / \rho, \\
d x^{*}=\rho^{-2} d x,
\end{gathered}
$$

a Ermakov-Ray-Reid type system is obtained, viz

$$
\begin{aligned}
& u_{x^{*} x^{*}}-\left[\Sigma_{k=1}^{4} c_{k}\left(u^{2}+v^{2}\right)^{k}-k_{\mathrm{III}}\right] u=\frac{k_{\mathrm{I}}^{2}}{u^{3}}+\frac{1}{u^{2} v} S\left(\frac{v}{u}\right), \\
& v_{x^{*} x^{*}}-\left[\Sigma_{k=1}^{4} c_{k}\left(u^{2}+v^{2}\right)^{k}-k_{\mathrm{III}}\right] v=\frac{k_{\mathrm{II}}}{v^{3}}+\frac{1}{v^{2} u} T\left(\frac{u}{v}\right),
\end{aligned}
$$

with invariant

$$
\mathscr{E}=\frac{1}{2}\left(v u_{x^{*}}-u v_{x^{*}}\right)^{2}+\int^{\xi=v / u} S(\xi) d \xi+\int^{\eta=u / v} T(\eta) d \eta+\frac{1}{2}\left[k_{\mathrm{I}}^{2}\left(\frac{v}{u}\right)^{2}+k_{\mathrm{II}}^{2}\left(\frac{u}{v}\right)^{2}\right] .
$$

If $S$ and $T$ admit the parametrisations in terms of $J(v / u)$ associated with underlying Hamiltonian structure so that

$$
S=2 \frac{v}{u} J(v / u)+\left(\frac{v}{u}\right)^{2} J^{\prime}(v / u) \quad, \quad T=-\left(\frac{v}{u}\right)^{2} J^{\prime}(v / u)
$$

then the system (2.28) admits a second invariant

$$
\mathscr{H}=\frac{1}{2}\left[u_{x^{*}}^{2}+v_{x^{*}}^{2}+\left(\frac{k_{\mathrm{I}}}{u}\right)^{2}+\left(\frac{k_{\mathrm{II}}}{v}\right)^{2}+k_{\mathrm{III}}\left(u^{2}+v^{2}\right)-\Sigma_{k=1}^{4} \frac{c_{k}}{k+1}\left(u^{2}+v^{2}\right)^{k+1}\right]+\frac{1}{u^{2}} J(v / u)
$$

while the Ermakov invariant relation (2.29) becomes

$$
\mathscr{E}=\frac{1}{2}\left(v u_{x^{*}}-u v_{x^{*}}\right)^{2}+\frac{1}{2}\left[k_{\mathrm{I}}^{2}\left(\frac{v}{u}\right)^{2}+k_{\mathrm{II}}^{2}\left(\frac{u}{v}\right)^{2}\right]+\left(\frac{u^{2}+v^{2}}{u^{2}}\right) J(v / u) .
$$

The identity

$$
\left(u^{2}+v^{2}\right)\left(u_{x^{*}}^{2}+v_{x^{*}}^{2}\right)-\left(v u_{x^{*}}-u v_{x^{*}}\right)^{2} \equiv\left(u u_{x^{*}}+v v_{x^{*}}\right)^{2},
$$

on use of the pair of invariants $\mathscr{E}$ and $\mathscr{H}$ now gives, 'in extenso',

$$
\begin{aligned}
\Sigma[2 \mathscr{H} & \left.-\left(\frac{k_{\mathrm{I}}}{u}\right)^{2}-\left(\frac{k_{\mathrm{II}}}{v}\right)^{2}-k_{\mathrm{III}} \Sigma-\frac{2}{u^{2}} J(v / u)+\Sigma_{k=1}^{4} \frac{c_{k}}{k+1} \Sigma^{k+1}\right] \\
& -2 \mathscr{E}+k_{\mathrm{I}}^{2}\left(\frac{v}{u}\right)^{2}+k_{\mathrm{II}}^{2}\left(\frac{u}{v}\right)^{2}+\frac{2}{u^{2}} \Sigma J(v / u)=\frac{1}{4} \Sigma_{x^{*}}^{2}
\end{aligned}
$$

where $\Sigma=u^{2}+v^{2}$. On reduction, (2.34) produces a nonlinear equation independent of $J(v / u)$ in $\Sigma$ alone, namely

$$
\Sigma\left[2 \mathscr{H}-k_{\mathrm{III}} \Sigma+\Sigma_{k=1}^{4} \frac{c_{k}}{k+1} \Sigma^{k+1}\right]-\left[2 \mathscr{E}+k_{\mathrm{I}}^{2}+k_{\mathrm{II}}^{2}\right]=\frac{1}{4} \Sigma_{x^{*}}^{2}
$$


Moreover, the Ermakov invariant relation (2.32), in turn, determines the ratio $\Lambda=v / u$ via the quadrature

$$
\int \frac{d\left(\tan ^{-1} \Lambda\right)}{\sqrt{2 \mathscr{E}-k_{\mathrm{I}}^{2} \Lambda^{2}-k_{\mathrm{II}}^{2} \Lambda^{-2}-2\left(1+\Lambda^{2}\right) J(\Lambda)}}= \pm X^{*}
$$

where

$$
d X^{*}=\Sigma^{-1} d x^{*} .
$$

Then,

$$
q_{1}= \pm \rho \sqrt{\frac{\Sigma}{1+\Lambda^{2}}} \quad, \quad q_{2}= \pm \rho \Lambda \sqrt{\frac{\Sigma}{1+\Lambda^{2}}}
$$

where $\rho$ is determined by the Ermakov equation (2.23). The $r_{k}(x), k=1,2$ are obtained by integration of the relations in (2.6).

\section{A Class of Jacobi Elliptic Solutions}

Here, by way of illustration, alignment of (2.35) is imposed with the nonlinear equation

$$
\Sigma_{x^{*}}^{2}+1-k^{2}+\left(k^{2}-2\right) \Sigma^{2}+\Sigma^{4}=0
$$

where $k$ here corresponds to the modulus of a Jacobi elliptic function. Thus, we set $\mathscr{H}=0$ together with $c_{1}=c_{3}=c_{4}=0$ and

$$
\begin{gathered}
2 \mathscr{E}+k_{\mathrm{I}}^{2}+k_{\mathrm{II}}^{2}=\frac{1-k^{2}}{4} \quad, \quad k_{\mathrm{III}}=\frac{k^{2}-2}{4}, \\
c_{2}=-\frac{3}{4} .
\end{gathered}
$$

The reduction (3.1) admits the particular solution

$$
\Sigma=\mathrm{dn}^{*}>0
$$

whence, the relation (2.37) yields

$$
X^{*}=\int \mathrm{nd}\left(\mathrm{x}^{*}, \mathrm{k}\right) \mathrm{dx}^{*}=\mathrm{k}^{\prime}-1 \cos ^{-1}\left(\mathrm{~cd}\left(\mathrm{x}^{*}, \mathrm{k}\right)\right),
$$

where $k^{\prime}=\sqrt{1-k^{2}}$ is the complementary modulus. 
If we proceed with the specialisation

$$
J(v / u)=\text { const } /\left[1+\left(\frac{v}{u}\right)^{2}\right]
$$

then (2.36) adopts the form

$$
\int \frac{d\left(\tan ^{-1} \Lambda\right)}{\sqrt{2 \overline{\mathscr{E}}-k_{\mathrm{I}}^{2}\left(\frac{v}{u}\right)^{2}-k_{\mathrm{II}}^{2}\left(\frac{v}{u}\right)^{-2}}}= \pm X^{*}
$$

where it is required that

$$
\overline{\mathscr{E}}>\frac{1}{2}\left[k_{\mathrm{I}}^{2}\left(\frac{v}{u}\right)^{2}+k_{\mathrm{II}}^{2}\left(\frac{v}{u}\right)^{-2}\right]>0 .
$$

Under the transformation

$$
u^{*}=-\frac{\mathbb{C}}{1+\left(\frac{v}{u}\right)^{2}},
$$

it is seen that, on taking the +ve sign in (3.6), the latter adopts the form

$$
\int \frac{d u^{*}}{\sqrt{\left(u^{*}-\lambda\right)\left(\mu-u^{*}\right)}}=2 \mathbb{C} X^{*}
$$

where

$$
\begin{gathered}
\lambda+\mu=2 \mathbb{C}^{-1}\left(\overline{\mathscr{E}}+k_{\mathrm{I}}^{2}\right) \quad, \quad \lambda \mu=k_{\mathrm{I}}^{2} \\
\mathbb{C}=-\sqrt{2 \overline{\mathscr{E}}+k_{\mathrm{I}}^{2}+k_{\mathrm{II}}^{2}} .
\end{gathered}
$$

On setting

$$
u^{*}=\lambda \cos ^{2} \theta+\mu \sin ^{2} \theta \quad, \quad \lambda, \mu>0,
$$

integration of (3.9) gives,

$$
\theta= \pm \mathbb{C} X^{*}
$$

up to an additive constant, whence

$$
u^{*}=\lambda+(\mu-\lambda) \sin ^{2} \mathbb{C} X^{*} .
$$

The ratio $\Lambda=v / u$ is thereby given in terms of $X^{*}$ via the relation (3.8). It can then be expressed in terms of $x^{*}$ by means of the relation (3.4).

It remains to determine $x^{*}$ in terms of the original spatial variable $x$ via the relation $(2.27)_{3}$ where $\rho$ is governed by the classical Ermakov equation (2.23). The latter admits a well-known nonlinear 
superposition principle readily established via Lie group methods (see e.g. [37,38]). Thus,

$$
\rho=\sqrt{a \rho_{1}^{2}+2 c \rho_{1} \rho_{2}+b \rho_{2}^{2}}
$$

where $\rho_{1}, \rho_{2}$ are linearly independent solutions of (2.12) and the constants $a, b$ and $c$ constrained by the relation

$$
a b-c^{2}=\frac{k_{\mathrm{III}}}{\mathscr{W}^{2}}
$$

where $\mathscr{W}\left(\rho_{1} ; \rho_{2}\right)=\rho_{1} \rho_{2 x}-\rho_{1 x} \rho_{2}$ is the Wronskian of $\rho_{1}, \rho_{2}$. Accordingly, here

$$
x^{*}=\int \frac{d x}{a \rho_{1}^{2}+2 c \rho_{1} \rho_{2}+b \rho_{2}^{2}} .
$$

In [48], in the context of Bloch wave propagation in optical lattices as described by a single component modulated cubic NLS equation, a periodic external potential $\Omega(x) \sim \cos 2 x$ was adopted in which case $\rho$ is given as a nonlinear superposition of Mathieu functions. If the external potential $\Omega$ is constant with $\mu-\Omega=\omega^{2}>0$ then the modulations as given by (2.24) of the coupled NLS system (2.1) are periodic with

$$
h_{k}(x)=\frac{c_{k}}{\left[a \cos ^{2} \omega x+2 c \cos \omega x \sin \omega x+b \sin ^{2} \omega x\right]^{2+k}} \quad, \quad k=1, \ldots, 4 .
$$

In general, a range of alignments of the nonlinear equation (2.35) in $\Sigma$ with canonical equations which admit Jacobi elliptic solutions may be made. In the present context, it is required that $\Sigma$ be positive. In that connection, in particular, it may be shown that the three-parameter class of nonlinear equations [9]

$$
\begin{aligned}
& \Sigma_{x^{*}}^{2}=-A_{0}^{2}-\left(3 b+k^{2}-2\right) \Sigma^{2}-\left[3 b^{2}+2\left(k^{2}-2\right) b+1-k^{2}\right] \frac{\Sigma^{4}}{A_{0}^{2}} \\
&-\left[b^{3}+\left(k^{2}-2\right) b^{2}+\left(1-k^{2}\right) b\right] \frac{\Sigma^{6}}{A_{0}^{4}}
\end{aligned}
$$

admits a positive, periodic solution

$$
\Sigma=\frac{A_{0}}{\sqrt{\mathrm{dn}^{2} \mathrm{x}^{*}+\mathrm{b}}} \quad, \quad A_{0}>0 .
$$

This result was exploited in [9] to isolate dark solitary pulse solutions of an unmodulatd single component nonic NLS equation. Therein, the parameters $A_{0}$ and $b$ were associated with the peak and trough of a wave train. In the present context of the modulated nonic NLS system (2.1), it is seen that alignment of (2.35) with (3.18) applies to a class with $c_{1}=c_{3}=0, c_{2} \neq 0, c_{4} \neq 0$.

\section{Modulated NLS Systems of Ermakov-Painlevé Type}

\subsection{A Ermakov-Painlevé II NLS System}

A hybrid NLS-Ermakov system with spatial modulation is introduced here of the type

$$
\begin{gathered}
i \Phi_{t}+\Phi_{x x}+\left[\frac{x}{2}+\varepsilon\left(|\Phi|^{2}+|\Psi|^{2}\right)\right] \Phi=\frac{1}{|\Phi|^{3}|\Psi|} S\left(\left|\frac{\Psi}{\Phi}\right|\right) \Phi \\
i \Psi_{t}+\Psi_{x x}+\left[\frac{x}{2}+\varepsilon\left(|\Phi|^{2}+|\Psi|^{2}\right)\right] \Psi=\frac{1}{|\Psi|^{3}|\Phi|} T\left(\left|\frac{\Phi}{\Psi}\right|\right) \Psi .
\end{gathered}
$$


By way of motivation, it is remarked that the single component similarly modulated cubic NLS equation

$$
i \sigma_{t}+\sigma_{x x}+\left(\delta x+\varepsilon|\sigma|^{2}\right) \sigma=0
$$

was shown by Chen and Liu in [8] to admit reduction to the canonical integrable NLS equation

$$
i \tau_{T}+\tau_{x x}+\varepsilon|\tau|^{2} \tau=0
$$

under the changes of variable

$$
\begin{gathered}
\sigma=\tau \exp i\left(\delta X T+(2 / 3) \delta^{2} T^{3}\right), \\
x=X+\delta T^{2}, \quad t=T .
\end{gathered}
$$

Thus, the single component modulated NLS equation (4.2) is amenable to methods of modern soliton theory such as the inverse scattering procedure as well as admitting geometric properties associated with invariance under a Bäcklund transformation with its concomitant nonlinear superposition principle. Here, the modulated two-component NLS system (4.1) is shown to admit symmetry reduction via (2.2) to an integrable hybrid Ermakov-Painlevé II system as recently set down in [39].

Thus, on introduction of the wave packet representations (2.2) into (4.1), a Ermakov-Painlevé II system results, namely

$$
\begin{aligned}
& q_{1 x x}+\left[\mu+\frac{x}{2}+\varepsilon\left(q_{1}^{2}+q_{2}^{2}\right)\right] q_{1}=\frac{1}{q_{1}^{2} q_{2}} S^{*}\left(q_{2} / q_{1}\right), \\
& q_{2 x x}+\left[\mu+\frac{x}{2}+\varepsilon\left(q_{1}^{2}+q_{2}^{2}\right)\right] q_{2}=\frac{1}{q_{1} q_{2}^{2}} T^{*}\left(q_{1} / q_{2}\right)
\end{aligned}
$$

where $S^{*}$ and $T^{*}$ are given by (2.8) and $r_{1}, r_{2}$ are determined by (2.6). Here, we proceed with the requirement that

$$
\frac{1}{q_{1}^{2} q_{2}} S^{*}\left(q_{2} / q_{1}\right)=\frac{\partial V}{\partial q_{1}} \quad, \quad \frac{1}{q_{1} q_{2}^{2}} T^{*}\left(q_{1} / q_{2}\right)=\frac{\partial V}{\partial q_{2}}
$$

in which case, the system (4.5) may be parametrised in terms of arbitrary $J\left(q_{2} / q_{1}\right)$ according to

$$
\begin{gathered}
q_{1 x x}+\left[\mu+\frac{x}{2}+\varepsilon\left(q_{1}^{2}+q_{2}^{2}\right)\right] q_{1}=\frac{2}{q_{1}^{3}} J\left(q_{2} / q_{1}\right)+\frac{q_{2}}{q_{1}^{4}} J^{\prime}\left(q_{2} / q_{1}\right), \\
q_{2 x x}+\left[\mu+\frac{x}{2}+\varepsilon\left(q_{1}^{2}+q_{2}^{2}\right)\right] q_{2}=-\frac{1}{q_{1}^{3}} J^{\prime}\left(q_{2} / q_{1}\right) .
\end{gathered}
$$

and admits the Ermakov invariant

$$
\mathscr{E}=\frac{1}{2}\left(q_{1} q_{2 x}-q_{2} q_{1 x}\right)^{2}+\left(\frac{q_{1}^{2}+q_{2}^{2}}{q_{1}^{2}}\right) J\left(q_{2} / q_{1}\right) .
$$

The identity

$$
\left(q_{1}^{2}+q_{2}^{2}\right)\left(q_{1 x}^{2}+q_{2 x}^{2}\right)-\left(q_{1} q_{2 x}-q_{2} q_{1 x}\right)^{2} \equiv\left(q_{1} q_{1 x}+q_{2} q_{2 x}\right)^{2}
$$

on use of (4.8), gives

$$
q_{1 x}^{2}+q_{2 x}^{2}-\frac{2 \mathscr{E}}{\Sigma}+\frac{2 J\left(q_{2} / q_{1}\right)}{q_{1}^{2}}=\frac{1}{4} \frac{\Sigma_{x}^{2}}{\Sigma}
$$


where $\Sigma=q_{1}^{2}+q_{2}^{2}$, whence

$$
q_{1 x} q_{1 x x}+q_{2 x} q_{2 x x}+\mathscr{E} \frac{\Sigma_{x}}{\Sigma^{2}}+d\left[\frac{J\left(q_{2} / q_{1}\right)}{q_{1}^{2}}\right] / d x=\frac{1}{4} \frac{\Sigma_{x} \Sigma_{x x}}{\Sigma}-\frac{1}{8} \frac{\Sigma_{x}^{3}}{\Sigma^{2}}=\frac{1}{2} \frac{\left(\Sigma^{1 / 2}\right)_{x x}}{\Sigma^{1 / 2}} \Sigma_{x} .
$$

The Ermakov-Painlevé II system (4.7) also shows that

$$
q_{1 x} q_{1 x}+q_{2 x} q_{2 x}+\left(\mu+\frac{x}{2}+\varepsilon\left(q_{1}^{2}+q_{2}^{2}\right)\right)\left(q_{1} q_{1 x}+q_{2} q_{2 x}\right)=d\left[\frac{J\left(q_{2} / q_{1}\right)}{q_{1}^{2}}\right] / d x
$$

and combination with (4.11) produces a single component Ermakov-Painlevé II equation in $\Sigma^{1 / 2}$, namely

$$
\left(\Sigma^{1 / 2}\right)_{x x}+\left(\mu+\frac{x}{2}+\varepsilon \Sigma\right) \Sigma^{1 / 2}=\frac{2 \mathscr{E}}{\Sigma^{3 / 2}} .
$$

In terms of $\Sigma$ and with the translation $z=2 \mu+x$, (4.13) delivers the canonical integrable Painlevé XXXIV equation

$$
\Sigma_{x x}-\frac{\Sigma_{z}^{2}}{2 \Sigma}+z \Sigma+2 \varepsilon \Sigma^{2}+\frac{(\alpha-\varepsilon / 2)^{2}}{2 \Sigma}=0
$$

with

$$
\mathscr{E}=-\frac{1}{8}(\alpha-\varepsilon / 2)^{2} \quad, \quad \varepsilon^{2}=1
$$

and where, in the present context, it is required that $\Sigma>0, J\left(q_{2} / q_{1}\right)<0$.

Interestingly, the importance of positive solutions of Painlevé XXXIV arises naturally elsewhere in connection with boundary value problems for a Painlevé II reduction of the classical two-ion Nernst-Planck system as described in [3]. Therein, the scaled electric field $E$ is governed by the canonical integrable Painlevé II equation

$$
E_{z z}=2 E^{3}+z E+\alpha
$$

while the associated ion concentrations $c_{ \pm}$are given by

$$
c_{ \pm}= \pm E_{z}+E^{2}+\frac{z}{2}
$$

The $c_{ \pm}$, which are necessarily positive, may be shown to be governed by Painlevé XXXIV. The link between the latter and Painlevé II has been previously established in the literature via a Hamiltonian representation for PII. Thus, each of the canonical Painlevé equations admits a Hamiltonian representation (see [23])

$$
\frac{\partial q}{\partial z}=\frac{\partial}{\partial p} H(p, q, z) \quad, \quad \frac{\partial p}{\partial z}=-\frac{\partial}{\partial q} H(p, q, z) .
$$

In particular, a Hamiltonian associated with PII is

$$
\mathscr{H}_{\text {II }}=\frac{1}{2} p^{2}-\left(q^{2}+\frac{z}{2}\right) p-\left(\alpha+\frac{1}{2}\right) q,
$$


whence

$$
\frac{d q}{d z}=p-q^{2}-\frac{z}{2} \quad, \quad \frac{d p}{d z}=2 p q+\alpha+\frac{1}{2} .
$$

Elimination of $p$ in (4.20) produces PII in $q$ while elimination of $q$ delivers PXXXIV in $p$. It is readily shown, more generally, that if $q$ is governed by PII then

$$
\Sigma=q_{z}-\varepsilon q^{2}-\varepsilon \frac{z}{2} \quad, \quad \varepsilon^{2}=1
$$

is a solution of PXXXIV as given by (4.14). In [3], this connection was used to generate sequences of exact solutions for the latter and hence the concentrations $c_{ \pm}$via the iterated action of the Bäcklund transformation due to Lukashevich [21] which is admitted by PII. The exact solutions so generated involve either Yablonski-Vorob'ev polynomials or classical Airy functions. The positivity constraints imposed on such solutions of PXXXIV were investigated in detail in [3]. These results carry over 'mutatis mutandis' in the present context of symmetry reduction via the wave packet representations (2.2) of the modulated NLS-Ermakov system (4.1). It is remarked that PXXXIV has also been recently derived in [40] in connection with integrable structure underlying the Korteweg capillarity system. Therein, it was recorded that the rational solutions of PXXXIV with $\alpha=n, n \in \mathbb{N}$ are given compactly by

$$
p_{n}(z)=\frac{r_{n+1}(z) r_{n-1}(z)}{2 r_{n}^{2}(z)}
$$

where the $r_{n}(z)$ are the Yablonskii-Vorob'ev polynomials determined by the quadratic recurrence relations $[11,45,47]$

$$
\begin{gathered}
r_{n+1} r_{n-1}=z r_{n}^{2}+4\left[r_{n, z}^{2}-r_{n} r_{n, z z}\right], \\
r_{-1}(z)=r_{0}(z)=1
\end{gathered}
$$

The analogous Airy-type solutions of PXXXIV corresponding to $\alpha=n-1 / 2$ are given by

$$
p_{n-1 / 2}(z)=\frac{s_{n+1}(z) s_{n-1}(z)}{2 s_{n}^{2}(z)}
$$

where the $s_{n}$ are given by the Toda-type recurrence relations

$$
\begin{aligned}
& s_{n+1} s_{n-1}=4\left[s_{n, z}^{2}-s_{n} s_{n, z z}\right], \\
& s_{0}(z)=1 \quad, \quad s_{1}(z)=\phi(z)
\end{aligned}
$$

with $\phi(z)$ given by the classical Airy equation

$$
\phi_{z z}+\frac{1}{2} z \phi=0
$$

With $\Sigma=q_{1}^{2}+q_{2}^{2}$ determined in terms of the $r_{n}(z)$ or $s_{n}(z)$, the ratio $\Lambda=q_{2} / q_{1}$ is given via the Ermakov invariant relation (4.8) with specified form of $J\left(q_{2} / q_{1}\right)$ and then

$$
q_{1}= \pm \sqrt{\frac{\Sigma}{1+\Lambda^{2}}} \quad, \quad q_{2}= \pm \Lambda \sqrt{\frac{\Sigma}{1+\Lambda^{2}}} .
$$


It is remarked that the class of Ermakov-Painlevé II systems with

$$
J\left(q_{2} / q_{1}\right)=\frac{\mathscr{E}}{1+\left(q_{2} / q_{1}\right)^{2}}-\frac{a^{2}}{4}\left(\frac{q_{1}^{2}-q_{2}^{2}}{q_{1}^{2}+q_{2}^{2}}\right)^{2 m}\left[1+\left(q_{1} / q_{2}\right)^{2}\right]
$$

has been recently discussed in [41]. A conventional Ermakov-Ray-Reid system with $J\left(q_{2} / q_{1}\right)$ of this type and $m=0$ arises in nonlinear optics in the variational analysis of the co-propagation of the optical pulses in a plane wave guide [28].

\subsection{A Ermakov-Painlevé IV NLS System}

Here, a hybrid NLS-Ermakov system incorporating a quadratic spatial modulation is introduced according to

$$
\begin{gathered}
i \Phi_{t}+\Phi_{x x}-\left[\frac{3}{4}\left(|\Phi|^{2}+|\Psi|^{2}\right)^{2}+2 x\left(|\Phi|^{2}+|\Psi|^{2}\right)+x^{2}-\zeta\right] \Phi=\frac{1}{|\Phi|^{3}|\Psi|} S\left(\left|\frac{\Psi}{\Phi}\right|\right) \Phi \\
i \Psi_{t}+\Psi_{x x}-\left[\frac{3}{4}\left(|\Phi|^{2}+|\Psi|^{2}\right)^{2}+2 x\left(|\Phi|^{2}+|\Psi|^{2}\right)+x^{2}-\zeta\right] \Psi=\frac{1}{|\Psi|^{3}|\Phi|} T\left(\left|\frac{\Phi}{\Psi}\right|\right) \Psi
\end{gathered}
$$

On insertion of the wave packet representations (2.2) into the above, one obtains the ErmakovPainlevé IV system

$$
\begin{aligned}
& q_{1 x x}-\left[\frac{3}{4}\left(q_{1}^{2}+q_{2}^{2}\right)^{2}+2 x\left(q_{1}^{2}+q_{2}^{2}\right)+x^{2}-\zeta-\mu\right] q_{1}=\frac{1}{q_{1}^{2} q_{2}} S^{*}\left(q_{2} / q_{1}\right) \\
& q_{2 x x}+\left[\frac{3}{4}\left(q_{1}^{2}+q_{2}^{2}\right)^{2}+2 x\left(q_{1}^{2}+q_{2}^{2}\right)+x^{2}-\zeta-\mu\right] q_{2}=\frac{1}{q_{1} q_{2}^{2}} T^{*}\left(q_{1} / q_{2}\right)
\end{aligned}
$$

where $S^{*}\left(q_{2} / q_{1}\right)$ and $T^{*}\left(q_{1} / q_{2}\right)$ are as given in (2.8) and the $r_{k}(x), k=1,2$ are to be obtained by integration of the relations (2.6).

Ermakov-Painlevé IV systems of the type (4.30) have been recently introduced in a more general setting in [39]. It is readily shown that, in this case, $\Sigma=q_{1}^{2}+q_{2}^{2}$ is governed by the canonical integrable Painlevé IV equation

$$
\Sigma_{x x}=\frac{1}{2} \frac{\Sigma_{x}^{2}}{\Sigma}+\frac{3}{2} \Sigma^{3}+4 x \Sigma^{2}+2\left(x^{2}-\alpha\right) \Sigma+\frac{\beta}{\Sigma}
$$

with $\alpha=\zeta+\mu$ and $\beta=4 \mathscr{E}$ where $\mathscr{E}$ is the Ermakov invariant given in the Hamiltonian case by the relation (4.8). The ratio $\Lambda=q_{2} / q_{1}$ is again determined via the Ermakov invariant relation and $q_{1}, q_{2}$ then by the relations in (4.27).

The diversity of Bäcklund and Schlesinger-type transformations admitted by Painlevë IV has been catalogued in the comprehensive work of [4]. With regard to the positivity constraint on $\Sigma$, the iterated action of a Bäcklund transformation on a seed bound state solution set down in [5] has recently been used in [42] to generate an infinite sequence of bound state solutions $\Sigma_{n}$ of Painlevé IV. These $\Sigma_{n}$ have regions separated by zeros on which they are positive. 


\section{References}

[1] J. Abdullaev, A.S. Desyatnikov and E.A. Ostravoskaya, Suppression of collapse for matter waves with orbital angular momentum, J. Opt. 13 (2011) 064023.

[2] D.W. Barclay, T.B. Moodie and C. Rogers, Cylindrical impact waves in inhomogeneous Maxwellian visco-elastic media, Acta Mechanica 29 (1978) 93-117.

[3] L.K. Bass, J.J.C. Nimmo, C. Rogers and W.K. Schief, Electrical structures of interfaces. A Painlevé II model, Proc. Roy. Soc. Lond. A466 (2010) 2117-2136.

[4] A.P. Bassom, P.A. Clarkson and A. C. Hicks, Bäcklund transformations and solution hierarchies for the fourth Painlevé equation, Stud. Appl. Math. 95 (1995) 1-71.

[5] A.P. Bassom, P.A. Clarkson, A. C. Hicks and J.B. McLeod, Integral equations and exact solutions for the fourth Painlevé equation, Proc. Roy. Soc. Lond. A437 (1992) 1-24.

[6] J. Belmonte-Beita, V.M. Pérez-Garcia and V. Vekslechik, Lie symmetries and solitons in nonlinear systems with spatially inhomogeneous nonlinearities, Physical Review Letters 98 (2007) 064102.

[7] J. Belmonte-Beita, V.M. Pérez-Garcia, V. Vekslechik and V.V. Konotop, Localised nonlinear waves in systems with time and space-modulated nonlinearities, Physical Review Letters 100 (2008) 164102.

[8] H.H. Chen and C.S. Liu, Solitons in non-uniform media, Phys. Rev. Lett. 37 (1976) 693-697.

[9] K.W. Chow and C. Rogers, Localized and periodic wave patterns for a nonic nonlinear Schrödinger equation, Phys. Lett. A 377 (2013) 2546-2550.

[10] P.L. Christiansen, J.C. Eilbeck, V.Z. Enolski and N.A. Kostov, Quasi-periodic solutions of the coupled nonlinear Schrödinger equations, Proc. Roy. Soc. London A 451 (1995) 685-700.

[11] P.A. Clarkson, Remarks on the Yablonskii-Vorob'ev polynomials, Phys. Lett. A 319 (2003) 137-144.

[12] D.L. Clements, C. Atkinson and C. Rogers, Antiplane crack problems for an inhomogeneous elastic material, Acta Mechanica 29 (1978) 199-211.

[13] D.L. Clements and C. Rogers, On the Bergman operator method and anti-plane contact problems involving an inhomogeneous half-space, SIAM J. Appl. Math. 34 (1978) 764-773.

[14] F. Cornolti, M. Lucchesi and B. Zambon, Elliptic Gaussian beam self-focussing in nonlinear media, Opt. Commun. 75 (1990) 129-135.

[15] A.S. Desyatnikov, D. Buccoliero, M.R. Dennis and Y.S. Kivshar, Suppression of collapse for spiral elliptic solitons, Phys. Rev. Lett. 104 (2010) 053902-1-053902-4.

[16] V.P. Ermakov, Second-order differential equations: conditions of complete integrability, Univ. Izy. Kiev 20 (1880) 1-25.

[17] A.M. Goncharenko, Y.A. Logvin, A.M. Samson, P.S. Shapovalev and S.I. Turovets Ermakov Hamiltonian systems in nonlinear optics of elliptic Gaussian beams, Phys. Lett. A 160 (1991) 138-142.

[18] A.M. Goncharenko, Y.A. Logvin, A.M. Samson and P.S. Shapovalov, Rotating ellipsoidal gaussian beams in nonlinear media, Opt. Commun. 81 (1991) 225-230.

[19] C.R. Guiliano, J.H. Marburger and A. Yariv, Enhancement of self-focussing threshold in sapphire with elliptical beams, Appl. Phys. Lett. 21 (1972) 58-60.

[20] F.C. Karal and J.B. Keller, Elastic wave propagation in homogeneous and inhomogeneous media, $J$. Acoust. Soc. America 31 (1959) 694-705.

[21] N.A. Lukashevich, The second Painlevé equation, Diff. Equations 7 (1971) 853-854.

[22] B.A. Malomed, Soliton Management in Periodic Systems (Springer, New York, 2006).

[23] K. Okamato, The Hamiltonians associated to the Painlevé equations, in The Painlevé Property: One Century Later, R. Conte (Ed), (Springer-Verlag, New York, 1999).

[24] J.R. Ray, Nonlinear superposition law for generalised Ermakov systems, Phys. Lett. A 78 (1980) 4-6.

[25] J.L. Reid and J.R. Ray, Ermakov systems, nonlinear superposition and solution of nonlinear equations of motion, J. Math. Phys. 21 (1980) 1583-1587.

[26] C. Rogers, C. Hoenselaers and J.R. Ray, On 2+1-dimensional Ermakov systems, J. Phys. A: Mathematical \& General 26 (1993) 2625-2633.

[27] C. Rogers, B. Malomed, K. Chow and H. An, Ermakov-Ray-Reid systems in nonlinear optics, J. Phys. A: Math. Theor. 43 (2010) 455214.

[28] C. Rogers, B. Malomed and H. An, Ermakov-Ray-Reid reductions of variational approximations in nonlinear optics, Stud. Appl. Math. 129 (2012) 389-413. 
[29] C. Rogers and H. An, Ermakov-Ray-Reid systems in 2+1-dimensional rotating shallow water theory, Stud. Appl. Math. 125 (2010) 275-299.

[30] C. Rogers and W.K. Schief, The pulsrodon in 2+1-dimensional magnetogasdynamics. Hamiltonian structure and integrability, J. Math. Phys. 52 (2011) 08371.

[31] C. Rogers and W.K. Schief, On the integrability of a Hamiltonian reduction of a 2+1-dimensional nonisothermal rotating gas cloud system, Nonlinearity 24 (2011) 3165-3178.

[32] C. Rogers, A novel Ermakov-Painlevé II system: n+1-dimensional coupled NLS and elastodynamic reductions, Stud. Appl. Math. 133 (2014) 214-231.

[33] C. Rogers, Hybrid Ermakov-Painlevé IV systems, J. Nonlinear Mathematical Physics 21 (2014) 628642.

[34] C. Rogers and W.K. Schief, Multi-component Ermakov systems: structure and linearization, J. Math. Anal. Appl. 198 (1996) 194-220.

[35] C. Rogers, Multi-component Ermakov and non-autonomous many-body systems connections, submitted (2017).

[36] C. Rogers, G. Saccomandi and L. Vergori, Ermakov-modulated nonlinear Schrödinger models: integrable reduction, J. Nonlinear Mathematical Physics 23 (2016) 108-126.

[37] C. Rogers and U. Ramgulam, A nonlinear superposition principle and Lie group invariance: application in rotating shallow water theory, Int. J. Nonlinear Mech. 24 (1989) 229-236.

[38] C. Rogers, W.K. Schief and P. Winternitz, Lie-theoretical generalization and discretization of the Pinney equation, J. Math. Anal. Appl. 216 (1997) 246-264.

[39] C. Rogers, On hybrid Ermakov-Painlevé systems. Integrable reduction, J. Nonlinear Mathematical Physics 24 (2017) 239-249.

[40] C. Rogers and P.A. Clarkson, Ermakov-Painlevé II symmetry reduction of a Korteweg capillarity system, Symmetry, Integrability and Geometry: Methods and Applications 13 (2017) 018.

[41] C. Rogers and W.K. Schief, On Ermakov-Painlevé II systems. Integrable reduction, Meccanica 51 (2016) 2967-2974.

[42] C. Rogers, A.P. Bassom and P.A. Clarkson, On integrable Ermakov-Painlevé IV systems, submitted (2017).

[43] W.K. Schief, H. An and C. Rogers, Universal and integrable aspects of an elliptic vortex representation in 2+1-dimensional magnetogasdynamics, Stud. Appl. Math. 130 (2013) 49-79.

[44] X.Y. Tang and P.K. Shukla, Solution of the one-dimensional spatially inhomogeneous cubic-quintic nonlinear Schrödinger equation with external potential, Phys. Rev. A 76 (2007) 013612.

[45] A.P. Vorob'ev, On the rational solutions of the second Painlevé equation, Diff. Equations 1 (1965) 7981.

[46] W.G. Wagner, H.A. Haus and J.H. Marburger, Large-scale self-trapping of optical beams in the paraxial ray approximation, Phys. Rev. 175 (1968) 256-266.

[47] A.I. Yablonskii, On rational solutions of the second Painlevé equation, Vesti AN BSSR, Ser. Fiz-Tech. Nauk 3 (1959) 30-35.

[48] J.F. Zhang, Yi S. Li, J. Meng, L. Wo and B.A. Malomed, Matter-wave solitons and finite-amplitude Bloch waves in optical lattices with a spatially modulated nonlinearity, Phys. Rev. A 82 (2010) 033614.

[49] W.P. Zhong, M.R. Belić, B.A. Malomed and T. Huang, Solitary waves in the nonlinear Schrödinger equation with Hermite-Gaussian modulation of the local nonlinearity, Phys. Rev. E 84 (2011) 046611.

[50] W.P. Zhong, M.R. Belić and T. Huang, Solitary waves in the nonlinear Schrödinger equation with spatially modulated Bessel nonlinearity, J. Opt. Soc. Am. B30 (2013) 1276-1283. 